\title{
用气溶胶飞行时间质谱实时探测甲苯光氧化产生 的单个二次有机气溶胶粒子*
}

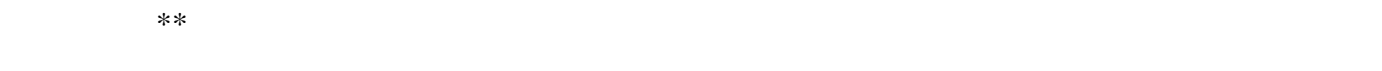

(中国科学院安徽光学精密机械研究所环境光谱学实验室, 合肥 230031)

\begin{abstract}
摘要 在烟雾腔内, 用紫外光照射甲苯、亚硝酸甲酯、一氧化氮和空气的混合物, 可以启动甲 苯和羟基自由基 $(\mathrm{OH} \cdot)$ 的光氧化反应和一系列的后续反应, 产生非挥发性和半挥发性有机化合物. 半挥发性有机化合物可以在气态和粒子态之间进行分配, 产生二次有机气溶胶粒子. 用自制的气 溶胶飞行时间质谱仪, 快速、实时测量这些粒子的尺度、它们的分子成分和直径分布.
\end{abstract}

\section{关键词甲苯 光氧化 二次有机气溶胶 气溶胶飞行时间质谱仪}

在城市上空的大气中, 芳香烃碳氢化合物主要 来源于汽车尾气和工业生产的排放物, 是挥发性有 机化合物的重要组成部分. 芳香烃碳氢化合物是城 市空气污染物, 它们的光化学过程可以产生臭氧和 二次有机气溶胶.气溶胶粒子影响大气的能见度、云 的形成和气候变化, 危害人类身体健康 ${ }^{[1 \sim 3]}$. 大气层 中的芳香烃碳氢化合物包括苯、甲苯、乙苯和二甲苯 等, 其中, 甲苯是最重要的芳香烃化合物 ${ }^{[4,5]}$, 它们在 都市二次有机气溶胶粒子形成过程中起到重要的支 配作用 ${ }^{[6]}$.

近年来，来源于甲苯光氧化的二次有机气溶胶 粒子的形成机理研究 ${ }^{[4,5,7 ~ 11]}$ 和探测方法研究 ${ }^{[4,7,12 ~ 14]}$ 已经引起人们的注意. 甲苯的光化学氧化是由羟基 自由基启动的. 在 $\mathrm{OH}-$ 甲苯反应中, 主要分为两步过
程: 提取反应和加成反应. 其中, 来自于甲基中 $\mathrm{H}$ 原 子提取反应只占 $10 \%$, 而到芳香环上的 $\mathrm{OH} \cdot$ 加成反应 占绝大部分 (大约 $90 \%)^{[7]}$. OH.- 甲苯反应以及它们的 后续反应可以生成种类繁多的非挥发性和挥发性有 机化合物. 半挥发性的有机化合物可以在气态和粒 子态之间进行分配，生成二次有机气溶胶粒子[4,7,9 11]. 二次气溶胶研究的一个重要内容就是测量单个气溶 胶粒子的直径和化学成分, 并给出大量粒子的直径 分布. 常规的方法是采用扫描电子移动光谱仪 (scanning electrical mobility spectrometers, SEMS)测 量粒子的直径, 配置凝结核计数器 (condensation nuclei counter, CNC) 测量粒子的数量 ${ }^{[10]}$. 而采用气相色 谱/质谱法分析二次有机气溶胶粒子的化学成分 ${ }^{[7,10]}$, 它利用过滤器收集二次有机气溶胶粒子, 用化学提 
取方法制备样品. 正的化学电离气相色谱离子俘获 质谱探测系统是近年来不断完善的探测二次有机气 溶胶粒子的方法 ${ }^{[4,12 ~ 14]}$. 这个探测系统可以分析比较 多的二次有机气溶胶粒子的化学组分, 如含羟基、羰 基和羧基官能团的组分以及多酮分子等. 另外, 作为 一种辅助手段, 傅立叶变换红外光谱技术(Fourier transform infrared spectroscope, FTIR)可以用来探测 二次有机气溶胶粒子化学组分中的官能团. 但是上 述方法都是分别测量粒子的直径和化学成分, 只能 统计得到大量粒子的直径和化学组分, 不能取得单 个气溶胶粒子的信息. 并且, 气相色谱/质谱方法和 化学电离气相色谱离子俘获质谱探测系统也存在一 些缺陷: 在样品收集过程中会引起二次反应或者半 挥发性有机化合物的损失; 在样品的化学提取和衍 生过程中, 消耗时间过长; 不能实时测量气溶胶粒子 的尺度和化学组分.

我们已经进行了二次有机气溶胶形成过程的理 论研究 ${ }^{[15]}$, 也进行了各种实验条件, 比如光照强度、 光照时间以及各种反应物浓度等对二次有机气溶胶 形成影响的实验研究 ${ }^{[16]}$. 本文主要介绍甲苯光氧化 产生的二次有机气溶胶粒子的探测和分析. 在实验 中, 采用自制的烟雾腔系统, 利用亚硝酸甲酯光解产 生的羟基自由基，启动甲苯的光氧化反应，模拟大气 中二次有机气溶胶的形成过程, 采用自制的气溶胶 飞行时间质谱仪(aerosol time of flight mass spectrometer, ATOFMS)测量二次有机气溶胶粒子. 用 ATOFMS分析二次有机气溶胶的过程中, 不需要制备 样品, 能够直接、实时和快速地测量气溶胶粒子, 同 时能够得到单个粒子的尺度和化学组分 ${ }^{[17 ~ 19]}$, 从而 得到气溶胶粒子的直径分布图. 从测量到的气溶胶 粒子的质谱, 得到气溶胶粒子各种化学成分, 在此以 前的测试方法并不具备这些优点.

\section{1 实验部分}

\section{1 实验装置}

甲苯的光氧化反应在我们自制的烟雾室内进行, 详细的实验装置已在文献 $[16,20]$ 进行了叙述, 在这 里只作简单介绍. 本实验装置由烟雾腔和配气管道
组成. 烟雾腔用石英管制成, 容积为 $23.3 \mathrm{~L}$, 表面积 体积比为 $22.4 \mathrm{~m}^{-1}$. 石英管的外边装有 16 只黑光灯, 每只功率为 $20 \mathrm{~W}$, 它所产生的辐射波长的范围为 300 420 nm. 配气管道的体积为 $0.84 \mathrm{~L}$, 上面安装一 个精密压力计, 指示范围为 10 5000 Pa, 能够准确显 示气体的压强.

空气压缩机产生的空气流经过 3 种过滤器：活性 炭、硅胶和 $\mathrm{DFU}^{\circledR}$ (Balston,USA), 依次除去空气中含 有的碳氢化合物、水分和悬浮颗粒物后变为洁净的空 气. 在每次实验前, 首先用洁净的空气吹扫烟雾腔 20 min. 然后采用机械洜抽真空至 $10^{-1} \mathrm{~Pa}$ 左右. 根据样 品配制要求, 用微量样品注射器将甲苯直接注射到 烟雾腔内. 根据配气装置的压力指示和反应物的浓 度比, 将亚硝酸甲酯和NO注入配气管道. 最后, 再 由干净的空气将它们吹入烟雾腔内. 在波长大于 300 $\mathrm{nm}$ 的光源照射下, 亚硝酸甲酯发生光解产生羟基自 由基 $(\mathrm{OH} \cdot)^{[21]}$, 它们反应过程如下:

$$
\begin{gathered}
\mathrm{CH}_{3} \mathrm{ONO}+h v(>300 \mathrm{~nm}) \rightarrow \mathrm{CH}_{3} \mathrm{O} \cdot+\mathrm{NO} \cdot \\
\mathrm{CH}_{3} \mathrm{O} \cdot+\mathrm{O}_{2} \rightarrow \mathrm{HCHO}+\mathrm{HO}_{2} \cdot \\
\mathrm{HO}_{2} \cdot+\mathrm{NO} \cdot \rightarrow \mathrm{OH} \cdot+\mathrm{NO}_{2} \cdot
\end{gathered}
$$

所有样品进样结束后, 继续在烟雾腔内注入干净空 气至一个大气压, 打开黑光灯, 开始光照反应.

\section{2 产物探测}

实验中，将亚硝酸甲酯(7249 $\mu \mathrm{L} / \mathrm{L})$ 、甲苯(98.2 $\mu \mathrm{L} / \mathrm{L}) 、 N O(2149 \mu \mathrm{L} / \mathrm{L})$ 和空气的混合物注入烟雾室中, 用两只 $20 \mathrm{~W}$ 的黑光灯照射 $1 \mathrm{~h}$. 然后, 将ATOFMS的 进样管道与烟雾室连接, 测量和分析由甲苯的光氧 化反应产生的二次有机气溶胶粒子. ATOFMS采用两 种不同的飞行时间技术，一种用于测量粒子直 径 [22], 另一种用于测量组成粒子的化学组分 ${ }^{[23]}$. ATOFMS由气溶胶进样系统、气溶胶粒子直径测量 系统、计时系统、直线式飞行时间质谱仪系统、四倍 频的Nd:YAG激光系统以及数据采集和处理系统等组 成 $[14,17 \sim 19,22,23]$.

在烟雾室中产生的二次有机气溶胶粒子, 通过 进样系统进入气溶胶粒子直径测量系统. 在直径测 量系统中, 气溶胶粒子穿过两束连续的激光, 产生的 散射信号转变成电信号, 传送到一个计时电路. 用计 
时电路可以得到测量粒子的飞行速度和空气动力学 直径. 计算机数据采集和处理系统根据气溶胶粒子 飞越两个散射光束之间的距离所用的时间，求出被 测粒子的直径. 根据气溶胶粒子的飞行速度和第二 个散射激光光束到气溶胶粒子电离中心的距离可以 求出气溶胶粒子飞越这段距离所用的时间，根据这 个时间可以确定触发脉冲式的 Nd:YAG 激光器的闪 光灯和调 $-\mathrm{Q}$ 开关的时间, 产生高功率的紫外激光脉 冲, 使气溶胶粒子发生脱吸附/电离. 此后, 不同质/ 荷比的离子进入直线式飞行时间质谱仪, 计算机通 过飞行时间数据采集器, 读取飞行时间, 进行质谱数 据采集. 与此同时, 计算机发出主动复位信号, 让计 时电路为跟踪下一个气溶胶粒子和确定电离激光器 的触发时间进行准备.

\section{2 结果和讨论}

根据 ATOFMS 的粒子直径测量系统、计时电路 和激光脱吸附/电离装置的设计原理, 它们的飞行时 间质谱只能来源于进行过直径测量的气溶胶粒子. 用我们自制的 ATOFMS 可以测量气溶胶粒子的直径、 化学成分和直径分布.

\section{1 单粒子的直径和化学成分}

图 1(a) (f)给出了采用 ATOFMS 测量到的 6 个单 个二次有机气溶胶粒子的飞行时间质谱图和它们的 直径大小. 这 6 个粒子是从大量的气溶胶粒子中挑选 出来的. 可以看到: 每一个气溶胶粒子对应于一个质 谱图, 每一个质谱都含有不同的质量与电荷比 $(\mathrm{m} / \mathrm{z})$, 每个 $m / z$ 又可能对应于不同的化学物质; 每个气溶剂 粒子的空气动力学直径也不相同. 上述结果说明: 每 个气溶胶粒子的直径和化学成分可能不同.

\section{2 直径分布图}

图 2 给出了 ATOFMS 测量的由甲苯光氧化产生 的气溶胶粒子的直径分布图. 它是大量单个二次有 机气溶胶粒子直径的统计分布图. 可以看出甲苯光 氧化产生的气溶胶粒子的最小直径为 $0.5 \mu \mathrm{m}$, 最大 直径为 $6.5 \mu \mathrm{m}$, 而大部分气溶胶粒子的直径分布在 $1.1 \sim 2.5 \mu \mathrm{m}$ 之间.

\section{3 产物的化学成分}

用ATOFMS测量空气中的气溶胶粒子的直径和 化学组分时, 每种化学组分的质量与电荷比的误差 可以为 $\pm 0.5^{[24,25]}$. 根据图 1 以及其他大量的单个气溶 胶粒子的质谱图, 可以总结分析出有机气溶胶粒子 的化学组分. 现在, 把部分质谱峰的 $m / z$ 的数据列举 如下: 36, 38, 40, 42, 48, 52, 54, 60, 64, 66, 68, 72, 74, 78, 84, 88, 90, 92, 103, 104, 105, 106, 118, 119, 120, $121,122,134,199$ 和 202 等. 这些不同 $m / z$ 的物质都对 应着不同的化合物分子. 其中, 我们把 $m / z=36,38,40$, 48, 52, 54 和 66 分别指认为 $\mathrm{C}_{3}, \mathrm{C}_{3} \mathrm{H}_{2}, \mathrm{C}_{3} \mathrm{H}_{4}, \mathrm{C}_{4}, \mathrm{C}_{4} \mathrm{H}_{4}$, $\mathrm{C}_{4} \mathrm{H}_{6}$ 和 $\mathrm{C}_{5} \mathrm{H}_{6}$ 碳氢化合物的碎片分子; $m / z=42$ 指认为 乙烯酮 $\left(\mathrm{C}_{2} \mathrm{H}_{2} \mathrm{O}\right)^{[26]} ; m / z=60$ 为乙醇醛 $\left(\mathrm{C}_{2} \mathrm{H}_{4} \mathrm{O}_{2}\right)^{[13]}$; $m / z=64$ 指认为呋喃 $\left(\mathrm{C}_{4} \mathrm{O}\right)^{[4]}$, 而 $m / z=68$ 指认为四氢呋 喃 $\left(\mathrm{C}_{4} \mathrm{H}_{4} \mathrm{O}\right)^{[26,27]} ; \mathrm{m} / \mathrm{z}=72$ 为甲基乙二醛 $\left(\mathrm{C}_{3} \mathrm{H}_{4} \mathrm{O}_{2}\right)^{[4]}$; $\mathrm{m} / \mathrm{z}=74$ 指认为乙醛酸 $\left(\mathrm{C}_{2} \mathrm{H}_{2} \mathrm{O}_{3}\right)$ 和羟基丙酮 $\left(\mathrm{C}_{3} \mathrm{H}_{6} \mathrm{O}_{2}\right)^{[4,13]} ; m / z=78$ 可为苯 $\left(\mathrm{C}_{6} \mathrm{H}_{6}\right) ; m / z=84$ 指认为 1,4-二氧代-2-丁烯 $\left(\mathrm{C}_{4} \mathrm{H}_{4} \mathrm{O}_{2}\right)^{[4]} ; \mathrm{m} / \mathrm{z}=88$ 为 2-羊基-1,3丙二醛和 2-氧代丙酸 $\left(\mathrm{C}_{3} \mathrm{H}_{4} \mathrm{O}_{3}\right)^{[4,12,13]} ; \mathrm{m} / \mathrm{z}=90$ 指认为 乙二酸 $\left(\mathrm{C}_{2} \mathrm{H}_{2} \mathrm{O}_{4}\right)^{[28]}$; 图中最强的信号 $m / z=92$ 应为母 体分子甲苯 $\left(\mathrm{C}_{6} \mathrm{H}_{5} \mathrm{CH}_{3}\right) ; m / z=104$ 可以指认为 2-羟基-

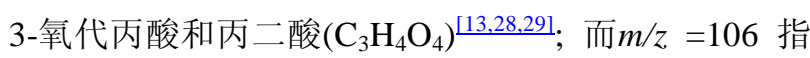

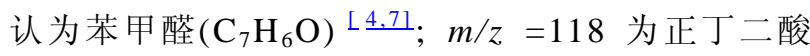
$\left(\mathrm{C}_{4} \mathrm{H}_{6} \mathrm{O}_{4}\right)^{[28,29]} ; m / z=120$ 则可以为间-甲基-苯甲醛(其 他两种同分异构体)和苯甲酩 $\left(\mathrm{C}_{8} \mathrm{H}_{8} \mathrm{O}\right)^{[13,26]} ; \mathrm{m} / \mathrm{z}=$ 121 为过氧乙酰硝酸酯 $\left(\mathrm{PAN}, \mathrm{C}_{2} \mathrm{H}_{3} \mathrm{O}_{5} \mathrm{~N}\right)^{[4]} ; \mathrm{m} / \mathrm{z}=122$ 可 以为苯甲酸、2-甲基-苯醌和 2-羟基-苯甲醛(其他两种 同分异构体 $)\left(\mathrm{C}_{7} \mathrm{H}_{6} \mathrm{O}_{2}\right)^{\left[{ }^{[4,7]} ;\right.} \mathrm{m} / \mathrm{z}=123$ 为硝基苯 $\left(\mathrm{C}_{6} \mathrm{H}_{5} \mathrm{NO}_{2}\right)^{[26]} ; \mathrm{m} / \mathrm{z}=134$ 可以指认为 2,3-二羟基-4-氧 代-丁酸 $\left(\mathrm{C}_{4} \mathrm{H}_{6} \mathrm{O}_{5}\right)^{[13]} \cdot m / z=119,199$ 和 202 表示了一些 未能指认的化合物. 上述分子是我们根据甲苯光氧 化可能的反应通道和一些文献结果，进行指认的部 分氧化产物. 这些结果表明: 用ATOFMS探测到的分 子中, 有些来自于甲苯的 $\mathrm{H}$-提取反应, 有些来自于 $\mathrm{OH}$.与甲苯加成反应及其后续反应. 这些化学成分 又可以分为芳香环保留的产物(如苯甲醛和苯甲酸)、 非芳香环保留的产物(呋喃和四氢呋喃), 以及环断裂 的含羰基产物(甲基乙二醛和 1,4-二氧代-2-丁烯). 在 我们的实验中, 所探测到的大部分化学成分与参考 

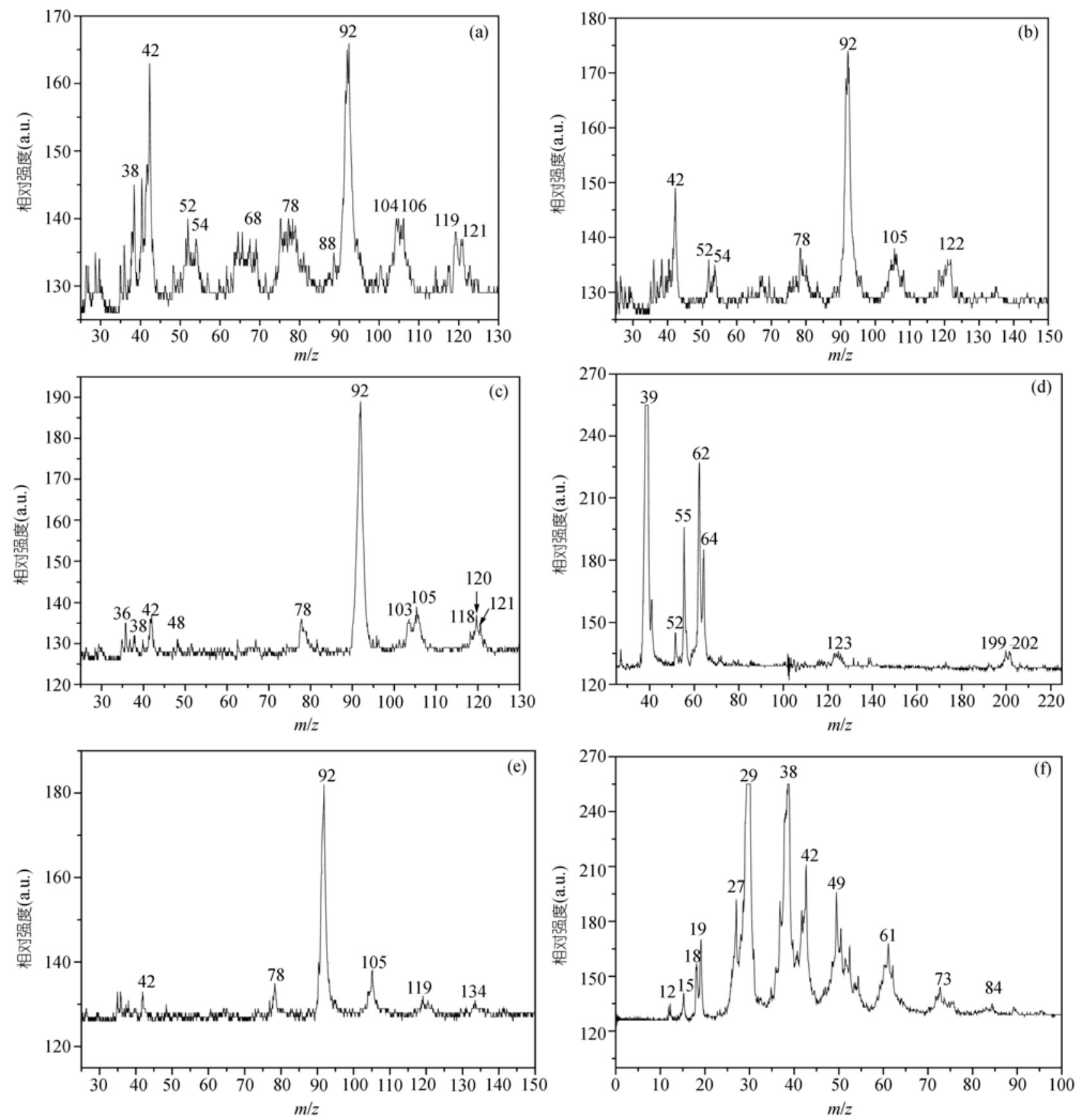

图 1 SOA 单个粒子的质谱图

空气动力学直径分别为: (a) $0.724 \mu \mathrm{m}$, (b) $1.982 \mu \mathrm{m}$, (c) $1.844 \mu \mathrm{m}$, (d) $1.597 \mu \mathrm{m}$, (e) $2.130 \mu \mathrm{m}$, (f) $1.982 \mu \mathrm{m}$

文献 $[4,7,12 \sim 14]$ 相同, 然而, 也有一些不同的化学成 分. 之所以产生这种结果, 是因为我们采用了不同的 探测方法. 事实上, 在大气环境中, 所有这些化学组 分是稳定的 ${ }^{[26 ~ 29]}$, 并且, 在燃烧过程中, 人们用激光
多光子电离和飞行时间质谱技术已经探测到它们 ${ }^{[26]}$. 例如, 四氢呋喃是一种持久性有机污染物, 它的分子 结构和特性已经被详细描述 ${ }^{[27]}$; 而在二次有机气溶 胶的化学组分中, 双羧酸 $\left(\mathrm{C}_{2} \sim \mathrm{C}_{9}\right)$ 是主要的种类之 


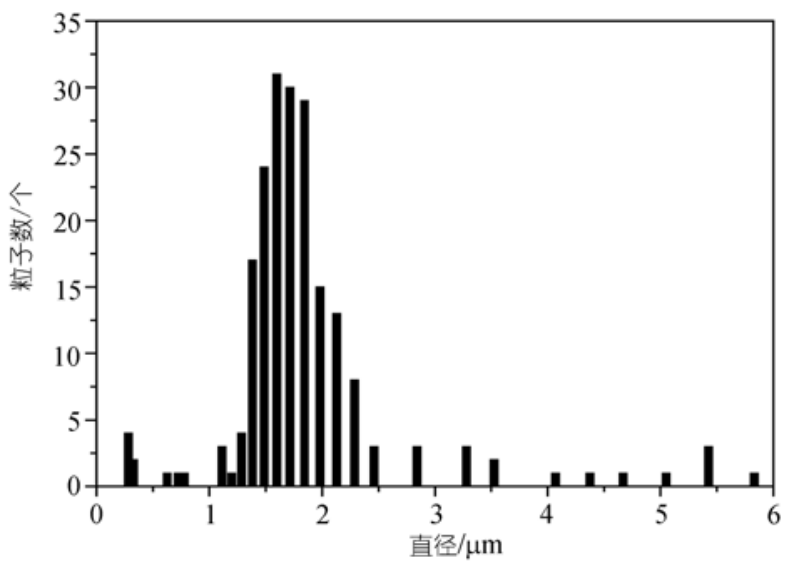

图 2 采用 ATOFMS 检测到的 SOA 粒子的直径分布

一28], 它包括HOOC-R-COOH 功能团, 此处 R 是正 烷基.

\section{3 结论}

采用烟雾腔模拟研究了大气中甲苯光氧化产生 二次有机气溶胶的化学过程, 采用实时测量气溶胶 粒子直径和化学成分的气溶胶飞行时间质谱, 测量 了二次有机气溶胶粒子的直径分布和分子成分. 通 过化学分析, 得到醛、酮和羧酸等重要的甲苯光氧化 产物, 为讨论甲苯光氧化反应的机理提供了新的信 息.

\section{参考文献}

1 Odum J R, Jungkamp T P W, Griffin R J, et al. The atmospheric aerosol-forming potential of whole gasoline vapor. Science, 1997, 276: 96 99[DOI]

2 Kalberer M, Paulsen D, Sax M, et al. Identification of polymers as major components of atmospheric organic aerosols. Science, 2004, 303: 1659 1662[DOI]

3 Zhang R Y, Suh I, Zhao J, et al. Atmospheric new particle formation enhanced by organic acids. Science, 2004, 304: 1487 1490[DOI]

4 Jang M, Kamens R M. Characterization of secondary aerosol from the photooxidation of toluene in the presence of $\mathrm{NO}_{x}$ and 1-proprene. Environ Sci Technol, 2001, 35: 3626 3639[DOI]

5 Suh I, Zhang R Y, Molina L T, et al. Oxidation mechnism of aromatic peroxy and bicylic radicals from $\mathrm{OH}$-toluene reactions. $\mathrm{J}$ Am Chem Soc, 2003, 122: 12655 12665[DOI]

6 Atkinson R. Atmospheric chemistry of VOCs and $\mathrm{NO}_{x}$. Atmos
Environ, 2000, 34: 2063 2101[DOI]

7 Forstner H J L, Flagan R C, Seinfeld J H. Secondary organic aerosol from the photooxidation of aromatic hydrocarbons: molecular composition. Environ Sci Technol, 1997, 31: 1345 1350[DOI]

8 Suh I, Zhang D, Zhang R Y, et al. Theoretical study of OH addition reaction to toluene. Chem Phys Lett, 2002, 364: 454 462[DOI]

9 Sato K, Klotz B, Hatakeyama S, et al. Secondary organic aerosol formation during the photo-oxidation of toluene: Dependence on initial hydrocarbon concentration. B Chem Soc Japan, 2004, 77(4): 667 671[DOI]

10 Odum J R, Hoffmann T, Bowman F M, et al. Gas/particle partitioning and secondary organic aerosol yields. Environ Sci Technol, 1996, 30: 2580 2585[DOI]

11 Stroud C A, Makar P A, Michelangell D V, et al. Simulating organic aerosol formation during the photooxidation of toluene/ $\mathrm{NO}_{x}$ mixtures: Comparing the equilibrium and kinetic assumption. Environ Sci Technol, 2004, 38: 1471 1479[DOI]

12 Edney O, Driscoll D J, Weathers W S, et al. Formation of polyketones in irradiated toluene/propylene/ $\mathrm{NO}_{x} /$ air mixtures. Aerosol Sci Technol, 2001, 35: 998 1008[DOI]

13 Kleindienst E, Conver T S, McIver C D, et al. Determination of secondary organic aerosol products from the photooxidation of toluene and their implications in ambient $\mathrm{PM}_{2.5}$. J Atmospheric Chemistry, 2004, 47: 79 100[DOI]

14 Jaoui M, Kleindienst T E, Lewandowski M, et al. Identification and quantification of aerosol polar oxygenated compounds bearing carboxylic or hydroxyl groups. 1. Method development. Anal Chem, 2004, 76: 4765 4778[DOI]

15 王振亚, 郝立庆, 张为俊. 二次有机气溶胶形成的化学过程. 化学进展, 2005, 17(4): 732 739

16 Hao L Q, Wang Z Y, Huang M Q, et al. The size distribution of the secondary organic aerosol particles from the photooxidation of toluene. J Environ Sci-China, 2005, 17(6): in press

17 Gard E, Mayer J E, Morrical B D, et al. Real-time analysis of individual atmospheric aerosol particles: Design and perfermance of a portable ATOFMS. Anal Chem, 1997, 69: 4083 4091[DOI]

18 Silva P J, Prather K A. Interpretation of mass spectra from organic compounds in aerosol time of flight mass spectrometry. Anal Chem, 2000, 72: 3553 3562[DOI]

19 Su Y, Sipin M F, Furutani H, et al. Development and characterization of an aerosol time-of-flight mass spectrometer with increased detection efficiency. Anal Chem, 2004, 76: 721 719

20 聂劲松, 秦 敏, 杨 勇, 等. 一种用于研究光化学反应烟雾 腔的结构和性能. 原子与分子物理学报, 2002, 19(2): 186 190 
21 Atkinson R, Carter W P L, Winer A M, et al. An experimental protocol for the determination of $\mathrm{OH}$ radical rate constants with organics using methyl nitrite photolysis as an $\mathrm{OH}$ radical source. J Air Pollution Control Association, 1981, 31: 1090 1092

22 夏柱红, 方 黎, 郑海洋, 等. 气溶胶单粒子粒径的实时测量 方法研究. 物理学报, 2004, 53(1): 320 324

23 夏柱红, 方 黎, 郑海洋, 等. 气溶胶单粒子化学成份的实时 测量. 分析化学, 2004, 32(7): 973 976

24 Liu D Y, Wenzel R J, Prather K A. Aerosol time of flight mass spectroscopy during the Atlanta Supersite Experiment: 1. Measurements. J Geophysical Research, 2003, 108, No. D7, SOS14: $1 \sim 16$

25 Wenzel R J, Liu D Y, Edgerton E S, et al. Aerosol time of flight mass spectroscopy during the Atlanta Supersite Experiment: 2. Scaling procedures. J Geophysical Research, 2003, 108, No. D7,
SOS15: 1 8

26 Grotheer H H, Nomayo M, Pokorny H, et al. Wavelength-resolved REMPI mass spectrometry for the monitoring of toxic incineration trace gases spectroscopic data and field measurements. Trands in Applied Spectroscopy, 2001, 3(3): 181 206

27 Ayres R U, Ayres L W. The life-cycle of chlorine, Part IV: Accounting for persistent cyclic organ-chlorines. J Industrial Ecology, 2000, 3(2): 121 159[DOI]

28 Nepotchatykh O V, Ariya P A. Degradation of dicarboxylic acids $\left(\mathrm{C}_{2}-\mathrm{C}_{9}\right)$ upon liquid-phase reactions with $\mathrm{O}_{3}$ and its atmospheric implications. Environ Sci Technol, 2002, 36: 3265 3269[DOI]

29 Bilde M, Svenningsson B, Monsteret J, et al. Even-odd alternation evaporation rates pressures of C3-C9 dicarboxylic acid aerosols. Environ Sci Technol, 2003, 37: 1371 1378[DOI] 